\section{Associação entre o grau de implantação da Rede Amamenta Brasil e indicadores de amamentação}

\author{
Association between the degree of implementation \\ of the Brazilian Breastfeeding Network and \\ breastfeeding indicators
}

Asociación entre el grado de implantación de la Red Amamanta Brasil e indicadores de lactancia materna
Sonia Isoyama Venancio 1

Elsa Regina Justo Giugliani 2

Osvaldinete Lopes de Oliveira Silva ${ }^{3}$

Juliana Stefanello 4

Maria Helena D'Aquino Benicio 4

Márcia Cristina Guerreiro dos Reis 5

Roberto Mario Silveira Issler 2

Lilian Cordova do Espírito Santo 2

Maria Regina Alves Cardoso 4

Gabriela Sintra Rios 1

\title{
Resumo
}

O objetivo foi avaliar a implantação da Rede Amamenta Brasil e seu impacto sobre indicadores de aleitamento materno (AM). Realizou-se análise de implantação de segundo tipo, incluindo 56 unidades básicas de saúde (UBS) de três municípios brasileiros. Para a avaliação do grau de implantação, criou-se um escore para cada UBS baseado no cumprimento dos critérios de certificação do Ministério da Saúde; para a análise dos efeitos da implantação, foram pesquisadas as prevalências de amamentação exclusiva e de amamentação. Verificou-se que 18 (32,1\%) UBS cumpriam os quatro critérios preconizados para certificação. Foram incluídas no estudo 1.052 crianças menores de um ano, sendo 563 menores de seis meses. Verificou-se que as UBS que cumpriam os quatro critérios de certificação tiveram maior prevalência de amamentação exclusiva (44\%), quando comparadas às demais UBS. Dificuldades para a implantação da Rede Amamenta Brasil foram identificadas, e os indicadores de AM variaram de acordo com o número de critérios de certificação cumpridos pelas UBS.

Aleitamento Materno; Atenção Primária à Saúde; Políticas Públicas de Saúde

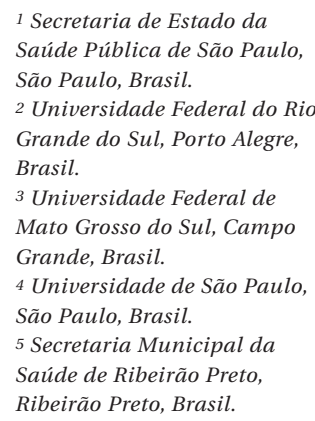

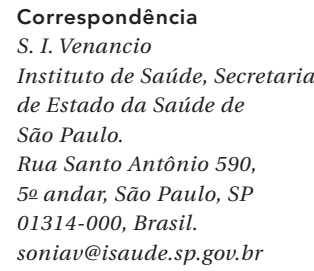




\section{Introdução}

Embora a prática do aleitamento materno (AM) esteja se expandindo no Brasil nas três últimas décadas 1, ela ainda está aquém das recomendações da Organização Mundial da Saúde (OMS) e do Ministério da Saúde, as quais preconizam a amamentação por dois anos ou mais, sendo de forma exclusiva nos primeiros seis meses de vida ${ }^{2}$.

Várias iniciativas em nível global têm se mostrado efetivas para aumentar as taxas de amamentação, tais como a Iniciativa Hospital Amigo da Criança 3,4 e diversos tipos de intervenções comunitárias, como programas desenvolvidos na Atenção Primária 5 , apoio de profissionais em casa ou nos serviços de saúde 6 , visitas domiciliares por profissionais de saúde 7 , aconselhamento por pares nos domicílios 8 e participação dos pais no apoio à amamentação 9 .

No Brasil, a partir da criação do Programa Nacional de Incentivo ao Aleitamento Materno no início da década de 1980, várias ações de promoção, proteção e apoio ao AM passaram a ser desenvolvidas. São exemplos: a elaboração e adoção da Norma Brasileira de Comercialização de Alimentos para Lactentes, Bicos, Chupetas e Mamadeiras (NBCAL), o desenvolvimento de uma ampla rede de bancos de leite humano e a implementação da Iniciativa Hospital Amigo da Criança 10. Contudo, o incentivo à amamentação na Atenção Básica permanecia sem uma política pública de âmbito nacional, havendo algumas experiências isoladas, como a da Secretaria Municipal de Saúde de Londrina, que lançou, em 1995, a Iniciativa Unidade Básica Amiga da Criança, e a da Secretaria de Estado de Saúde do Rio de Janeiro, que idealizou, em 1999, a Iniciativa Unidade Básica Amiga da Amamentação 11.

Nesse contexto, o Ministério da Saúde lançou, em 2008, uma estratégia nacional de promoção, proteção e apoio ao AM na Atenção Básica, denominada Rede Amamenta Brasil, atualmente denominada Estratégia Amamenta e Alimenta Brasil, após integração com a Estratégia Nacional de Promoção da Alimentação Complementar Saudável (ENPACS), em 2011. A proposta alinhava-se com a Política de Educação Permanente, tendo como referencial a metodologia crítico-reflexiva, com foco na discussão das equipes de Atenção Básica sobre as implicações de seu processo de trabalho na promoção, proteção e apoio ao AM 11. O processo de implantação da Rede Amamenta Brasil nas unidades básicas de saúde (UBS) iniciava por meio da realização de uma oficina de trabalho, com duração de seis horas, com toda a equipe da UBS. Contava com a participação de pelo menos um profissional de cada categoria funcional, incluindo administrativos e terceirizados, durante a qual era discutido o processo de trabalho em relação às ações de promoção, proteção e apoio ao AM, expondo-se dificuldades e pactuando-se ações em busca de soluções a partir da realidade local. Após a realização dessa oficina, a UBS deveria ser acompanhada por um tutor da Rede, que tinha a função de apoiar e monitorar as ações de promoção, proteção e apoio ao AM dessa unidade. O Ministério da Saúde considerava aptas para receber a certificação na Rede as unidades que cumprissem os seguintes critérios: participação de no mínimo $80 \%$ da equipe na oficina de trabalho; monitoramento contínuo dos indicadores de AM em sua área de abrangência; concretização de pelo menos uma ação pactuada na oficina; e implementação de fluxograma de atendimento à dupla mãe-bebê no período de amamentação 11 .

A implantação da Rede Amamenta Brasil no nível nacional ocorreu de forma gradativa, com a adesão de diversos estados e municípios em diferentes regiões do Brasil 11. Durante esse processo, surgiu a necessidade de compreender melhor os mecanismos envolvidos após a adesão inicial dos estados, identificando aspectos que contribuíam para o fortalecimento e qualificação da intervenção, ou que dificultavam o processo. Nesse sentido, realizou-se uma pesquisa avaliativa utilizando o referencial da análise de implantação do componente 1 , na qual se observou a variação do contexto organizacional e sua relação com o grau de implantação. Os resultados comprovaram que os três municípios analisados se encontravam em diferentes graus de implantação da Rede Amamenta Brasil e que contextos mais favoráveis a esse processo (apoio da gestão municipal, alocação de recursos para capacitação em manejo do AM e papéis fortalecidos do coordenador técnico da Rede e dos tutores) estavam relacionados a graus de implantação mais avançados 12 .

Posteriormente, para responder se a Rede Amamenta Brasil tinha impacto sobre os indicadores de AM, considerou-se oportuna a realização de uma avaliação da implantação em seu segundo componente, ou seja, a análise da relação entre diferentes graus de implantação da Rede Amamenta Brasil e alguns indicadores de AM em crianças menores de um ano de vida. Este artigo apresenta os resultados dessa avaliação.

\section{Métodos}

Realizou-se pesquisa avaliativa do tipo análise de implantação em seu segundo componente 13 (análise das variações da implantação nos 
efeitos observados). A pesquisa compreendeu a análise do grau de implantação da Rede Amamenta Brasil em UBS de três municípios e sua influência sobre a situação da amamentação em crianças menores de um ano de idade atendidas nessas unidades.

Para a seleção dos municípios, primeiramente foram identificados aqueles que possuíam UBS certificadas na Rede Amamenta Brasil e que dispunham de dados de base sobre a prevalência do AM. Dentre esses municípios, foram selecionados três (Dourados, Mato Grosso do Sul; Porto Alegre, Rio Grande do Sul; e Ribeirão Preto, São Paulo) com características distintas quanto à sua localização (macrorregião), tamanho e cobertura da Estratégia Saúde da Família (ESF), porém com indicadores de AM não muito discrepantes.

Dourados, contava com população de 196.035 mil habitantes e cobertura da ESF de 83\%. Porto Alegre, capital do Rio Grande do Sul, era com 1.409.351 habitantes e menos da metade da população é coberta pela ESF (45\%). O terceiro Município foi Ribeirão Preto, com população de 604.682 habitantes e cobertura da ESF de 14,2\%. A prevalência de amamentação exclusiva em menores de seis meses, em 2008, foi $35 \%$ em Dourados e $41 \%$ em Porto Alegre e Ribeirão Preto 14 .

Em cada município, foi selecionada uma amostra intencional de UBS representando três situações distintas em relação à Rede Amamenta Brasil: UBS certificadas na Rede Amamenta Brasil (UBS/Certificada), UBS que haviam realizado oficina da Rede, mas que ainda não haviam sido certificadas no momento do estudo (UBS/Oficina) e UBS que não tinham nenhum envolvimento com a Rede (UBS/Controle). Foram incluídas todas as UBS certificadas em cada município. Para a seleção das UBS/Oficina e UBS/Controle, levaram-se em consideração alguns critérios, como localização, número de crianças atendidas/ mês e modelo de atenção (ESF ou UBS tradicional), a fim de incluir no estudo UBS com características semelhantes, exceto pela adesão ou certificação na Rede Amamenta Brasil. Em Dourados, no entanto, não foi possível a inclusão de UBS/ Controle, uma vez que todas as UBS já haviam realizado a oficina da Rede. Sendo assim, Ribeirão Preto e Porto Alegre incluíram um número maior de crianças nas UBS controle para fins de equivalência com as demais categorias.

Para caracterizar as UBS, foi construído um instrumento para coleta de dados contendo questões sobre o cumprimento dos critérios de certificação da Rede Amamenta Brasil e características da implantação dessa estratégia. As entrevistas foram aplicadas aos gerentes na própria UBS, com duração aproximada de 40 minutos.
Para a avaliação do grau de implantação da Rede Amamenta Brasil, realizou-se uma avaliação normativa, utilizando-se os critérios estabelecidos pelo Ministèrio da Saúde 11 para certificação das UBS. Os escores para cada UBS foram construídos com base nas respostas dos gerentes quanto ao cumprimento dos critérios, sendo o menor valor 0 (nenhum critério atendido) e o maior valor 4 (todos os critérios atendidos).

Para a análise dos efeitos da implantação da Rede Amamenta Brasil, pesquisou-se a prática do AM em crianças menores de um ano de idade, atendidas nas UBS selecionadas nos três municípios. Tomando-se por base para o cálculo amostral um nível de $95 \%$ de confiança, poder do teste de $80 \%$, relação entre expostos e não expostos à intervenção de 1:1 e um aumento esperado de $25 \%$ na prevalência de aleitamento materno exclusivo (AME) em menores de seis meses com a intervenção, foram incluídas na amostra 300 crianças em cada município. Para que houvesse equilíbrio com relação à idade das crianças, foi preestabelecido que elas deveriam ser distribuídas igualmente em quatro faixas etárias: 0-2,9 meses; 3-5,9 meses; 6-8,9 meses e 9-11,9 meses. Para a obtenção dos dados sobre a prática do AM, foram entrevistadas mães selecionadas de forma aleatória por ocasião do atendimento de puericultura de seus filhos nas UBS incluídas no estudo, até que se completasse o número de crianças definido para cada faixa etária em cada unidade. Em algumas UBS menores, em que os atendimentos às crianças em algumas faixas etárias eram reduzidos, foi realizada busca ativa nos domicílios. Para a realização dessa busca, utilizaram-se os registros das unidades, sendo as crianças selecionadas de forma aleatória.

O questionário aplicado às mães continha perguntas sobre alimentação das crianças nas 24 horas anteriores à entrevista, conforme preconizado pela OMS ${ }^{15}$. Os indicadores de AM utilizados foram AME em menores de seis meses e continuidade da amamentação em crianças de 9-12 meses, conforme proposto em pesquisas nacionais 14 .

A coleta de dados foi realizada entre outubro de 2012 e março de 2013 por entrevistadores previamente treinados.

A análise dos dados partiu do modelo teórico apresentado na Figura 1. Todas as análises descritivas foram realizadas por meio do programa SPSS 13.0 (SPSS Inc., Chicago, Estados Unidos), e as figuras foram confeccionadas no programa Excel (Microsoft Corp., Estados Unidos). A análise da associação entre o escore de implantação da Rede Amamenta Brasil e os indicadores de AM foi realizada mediante o teste do qui-quadrado a um nível de $5 \%$ de significância . 


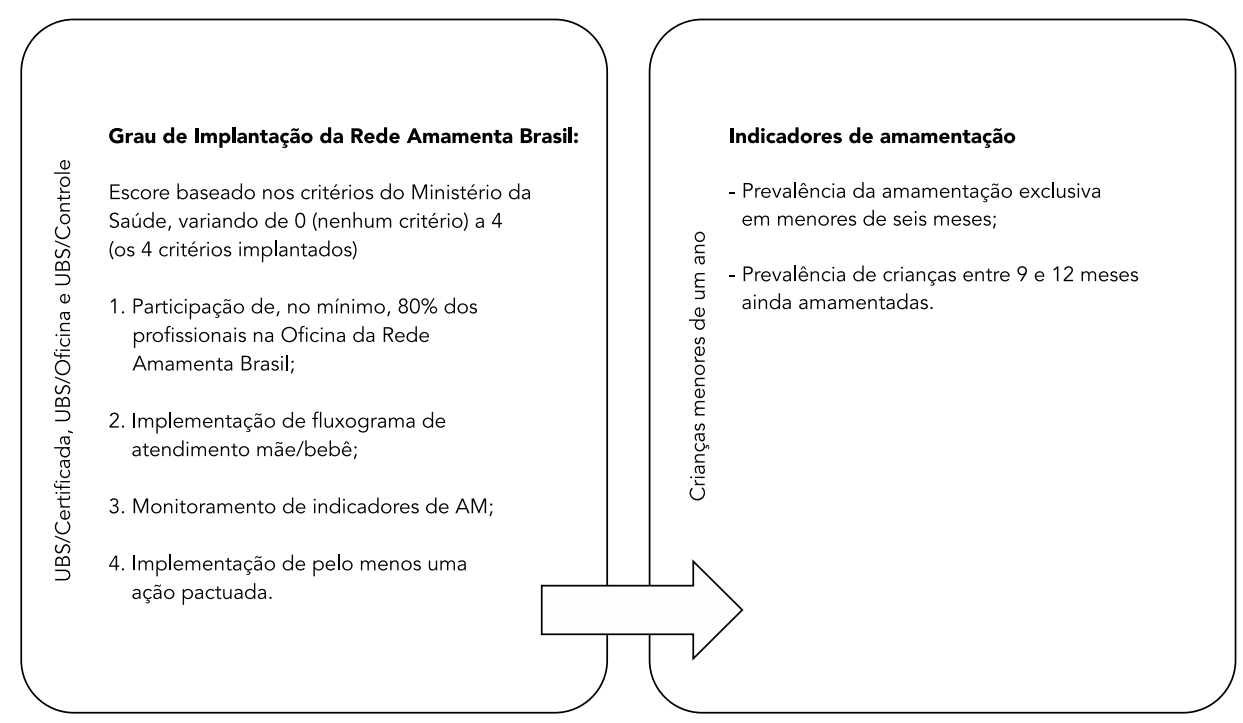

AM: aleitamento materno; UBS: unidades básicas de saúde.

O projeto foi aprovado pelo Comitê de Ética do Instituto de Saúde da Secretaria de Estado da Saúde de São Paulo sob o parecer no 95.738 e pelo Comitê de Ética em Pesquisa da Secretaria Municipal de Saúde de Porto Alegre no 001.037827.12.0.

\section{Resultados}

Foram incluídas no estudo 56 UBS, sendo 22 UBS/ Certificadas, 20 UBS/Oficina e 14 UBS/Controle. A Tabela 1 apresenta a caracterização das UBS segundo o cumprimento dos critérios definidos pelo Ministério da Saúde para certificação, bem como o detalhamento de ações relacionadas à implantação da Rede Amamenta Brasil.

De forma geral, verifica-se que as UBS certificadas apresentam melhor desempenho nas ações previstas pela Rede Amamenta Brasil em comparação às UBS que haviam realizado a oficina de trabalho, sem, no entanto, terem sido certificadas até o momento das entrevistas. Em contrapartida, observa-se também que algumas UBS certificadas, as quais, portanto, deveriam cumprir todos os critérios de certificação, deixaram de atender esses critérios segundo o relato dos gerentes. Chama a atenção que, mesmo entre as UBS/Certificada, identificaram-se falhas na utilização do fluxograma de atendimento à du- pla mãe-bebê, acolhimento da demanda espontânea, estabelecimento de fluxo de referência para atendimento de casos graves, utilização de protocolo para manejo dos problemas de amamentação e monitoramento dos indicadores de AM. Por outro lado, os gerentes de algumas UBS do grupo controle relataram a implantação de algumas ações previstas pela Rede Amamenta Brasil. Por fim, outro dado que merece destaque é o baixo cumprimento da recomendação do Ministério da Saúde de realização de visitas periódicas às UBS pelos tutores da Rede Amamenta Brasil.

Quando atribuído o escore do grau de implantação da Rede Amamenta Brasil, verificouse que sete $(12,5 \%)$ UBS não cumpriam critério algum de certificação, 17 (30,4\%) cumpriam um critério, seis $(10,7 \%)$ cumpriam dois critérios, oito $(14,3 \%)$ cumpriam três critérios e 18 $(32,1 \%)$ cumpriam os quatro critérios de acordo com o preconizado pelo Ministério da Saúde para certificação.

Na Figura 2, confronta-se o escore de implantação da Rede Amamenta Brasil e o tipo de UBS (Certificada, com Oficina e Controle). Verificase que, entre as UBS/Certificada, somente metade cumpria os quatro critérios avaliados pelo Ministério da Saúde para a certificação na Rede Amamenta Brasil; entre as UBS/Oficina, 15\% não cumpriam nenhum dos critérios de certificação e 
Tabela 1

Caracterização das UBS segundo cumprimento dos critérios definidos pelo Ministério da Saúde para certificação e detalhamento de ações relacionadas à implantação da Rede Amamenta Brasil. Dourados (Mato Grasso do Sul), Porto Alegre (Rio Grande do Sul) e Ribeirão Preto (São Paulo), Brasil, 2012.

\begin{tabular}{|c|c|c|c|c|}
\hline \multirow[t]{2}{*}{ Variáveis } & \multicolumn{3}{|c|}{ Perfil da UBS } & Total (n) \\
\hline & $\begin{array}{c}\text { UBS/Certificada } \\
\text { (\%) }[n=22]\end{array}$ & $\begin{array}{l}\text { UBS/Oficina } \\
(\%)[n=20]\end{array}$ & $\begin{array}{l}\text { UBS/Controle } \\
\text { (\%) }[n=14]\end{array}$ & {$[n=56]$} \\
\hline
\end{tabular}

\section{Critério 1}

Participação de no mínimo 80\% da equipe na Oficina *

\section{Critério 2}

Adoção de fluxograma *

Utilização de fluxograma **

\section{Sempre}

Na maioria das vezes

Às vezes

Fluxograma facilmente acessível **

Atendimento às mães que procuram a UBS por problema de AM sem consulta

agendada

Sempre

Na maioria das vezes

Fluxo de referência estabelecido

Adoção de protocolo para manejo dos problemas de AM

\section{Critério 3}

Realização de monitoramento dos indicadores de $\mathrm{AM}$ *

Plenamente

Parcialmente

Não

Sistema utilizado para o monitoramento ***

SISVAN web

Planilhas do Ministério da Saúde

Outros

Compartilhamento dos resultados do monitoramento ***

\section{Critério 4}

Existência de planejamento para as ações de AM

Implantação de pelo menos uma proposta do Plano de Ação \#

Atuação do tutor

Visitas regulares do tutor às UBS

Frequência das visitas dos tutores às UBS

Bimestral

Trimestral

Outros

Mudanças nas práticas de incentivo ao AM após a implantação da Rede

Amamenta Brasil

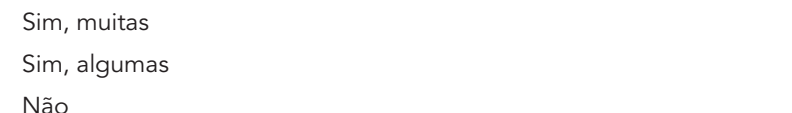

\section{0,0}

100,0

68,2

53,3

33,3

13,3

80,0

90,9

9,1

90,9

63,6

86,4

13,6

-

25,0

50,0

25,0

61,9

72,7

94,1

61,9

30,8

53,8

15,4

27,3

63,6

9,1
40

37,5

50,0

12,5

62,5

100,0

-

80,0

45,0

70,0

20,0

10,0

11,8

41,2

47,1

47,4

55,0

90,9

50,0

22,2

55,6

22,2
28

35,7

80,0

20,0

92,9

7,1

85,7

78,6

21,4

28,6

50,0

$28,6 \quad 9$

$14,3 \quad 18$

$57,1 \quad 17$

$71,4 \quad 27$

M: aleitamento materno; SISVAN: Sistema de Vigilância Alimentar Nutricional; UBS: unidades básicas de saúde.

* Itens definidos pelo Ministério da Saúde para certificação, considerados para a construção do escore do grau de implantação da Rede Amamenta Brasil;

** Consideradas apenas as UBS que adotavam fluxograma;

*** Consideradas apenas as UBS que realizavam monitoramento;

\# Consideradas apenas as UBS que tinham Plano de Ação. 
Comparação entre o tipo de UBS e escore de implantação da Rede Amamenta Brasil. Dourados (Mato Grosso do Sul), Porto Alegre (Rio Grande do Sul) e Ribeirão Preto (São Paulo), Brasil, 2012.

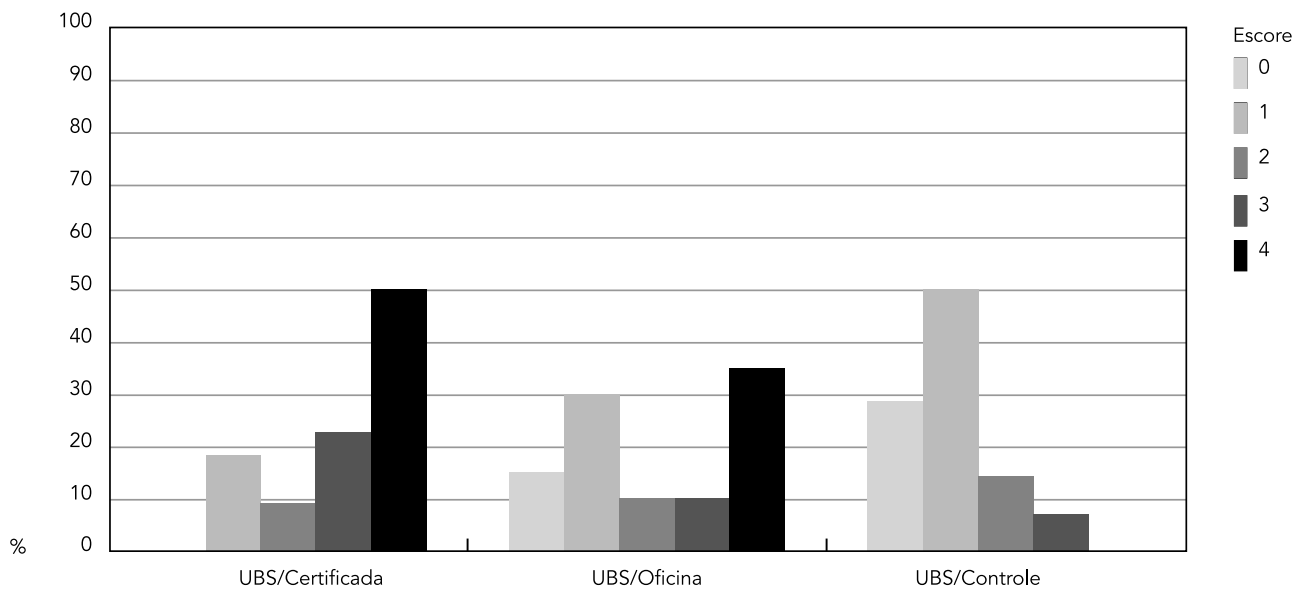

UBS: unidades básicas de saúde.

$35 \%$ já cumpriam os quatro critérios; finalmente, entre as UBS/Controle, contrariando a expectativa, somente $28,6 \%$ não cumpriam critério algum de certificação da Rede Amamenta Brasil.

Para a análise dos indicadores de AM, foram incluídas no estudo 1.052 crianças menores de um ano, sendo 563 menores de seis meses. Em relação às características maternas, a maioria das mães relatou idade entre 21 e 35 anos (67\%), era de cor branca (44\%) e multípara (57\%); 35\% tinham entre 9 e 12 anos de escolaridade. Todas relataram acompanhamento pré-natal, $61 \%$ tiveram parto vaginal e $64 \%$ referiram que a criança foi atendida pela equipe da UBS na primeira semana de vida. Metade das crianças era do sexo masculino e $8,3 \%$ tiveram baixo peso ao nascer. Verificou-se que $38 \%$ das crianças menores de seis meses estavam em amamentação exclusiva e 56,4\% das crianças entre 9 e 12 meses de vida continuavam sendo amamentadas.

A Figura 3 mostra as prevalências de amamentação exclusiva em menores de seis meses e de amamentação em crianças de 9-12 meses, segundo o escore de implantação da Rede Amamenta Brasil. Verifica-se que as UBS cujo escore apontou cumprimento dos quatro critérios de certificação na Rede Amamenta Brasil tiveram maior prevalência de AME quando comparadas às demais UBS. O mesmo não se verifica em relação à manutenção da amamentação, uma vez que a maior prevalência do AM em crianças de
9-12 meses ocorreu entre as UBS que cumpriam dois critérios de certificação. Para a verificação da existência de associação estatisticamente significativa entre o escore de implantação da Rede Amamenta Brasil e as prevalências de AME e AM, as UBS foram agrupadas segundo três categorias (nenhum critério de certificação; um, dois ou três critérios de certificação; e quatro critérios de certificação). As prevalências de AME em menores de seis meses foram 28,6\%, 35,6\% e $44,4 \%$, enquanto as prevalências de AM nas crianças entre 9 e 12 meses foram 45,4\%, 58,8\% e $59,6 \%$, respectivamente. Observou-se tendência de aumento da prevalência do AME em menores de seis meses com o aumento dos critérios de certificação $(p=0,07)$. A mesma tendência não ocorreu em relação à amamentação em crianças de 9-12 meses. Nesse indicador, o melhor desempenho ocorreu entre as UBS que cumpriam dois critérios de certificação.

\section{Discussão}

Inicialmente, vale a pena discutir os resultados relacionados à implantação da Rede Amamenta Brasil nas UBS estudadas. Identificou-se que o cumprimento dos quatro critérios utilizados pelo Ministério da Saúde pode não ter continuidade após a certificação e, consequentemente, as UBS certificadas podem não ter os atributos 
Prevalência de amamentação exclusiva em menores de seis meses e de AM em crianças entre 9 e 12 meses, segundo grau de implantação da Rede Amamenta Brasil. Dourados (Mato Grasso do Sul), Porto Alegre (Rio Grande do Sul) e Ribeirão Preto (São Paulo), Brasil, 2012.

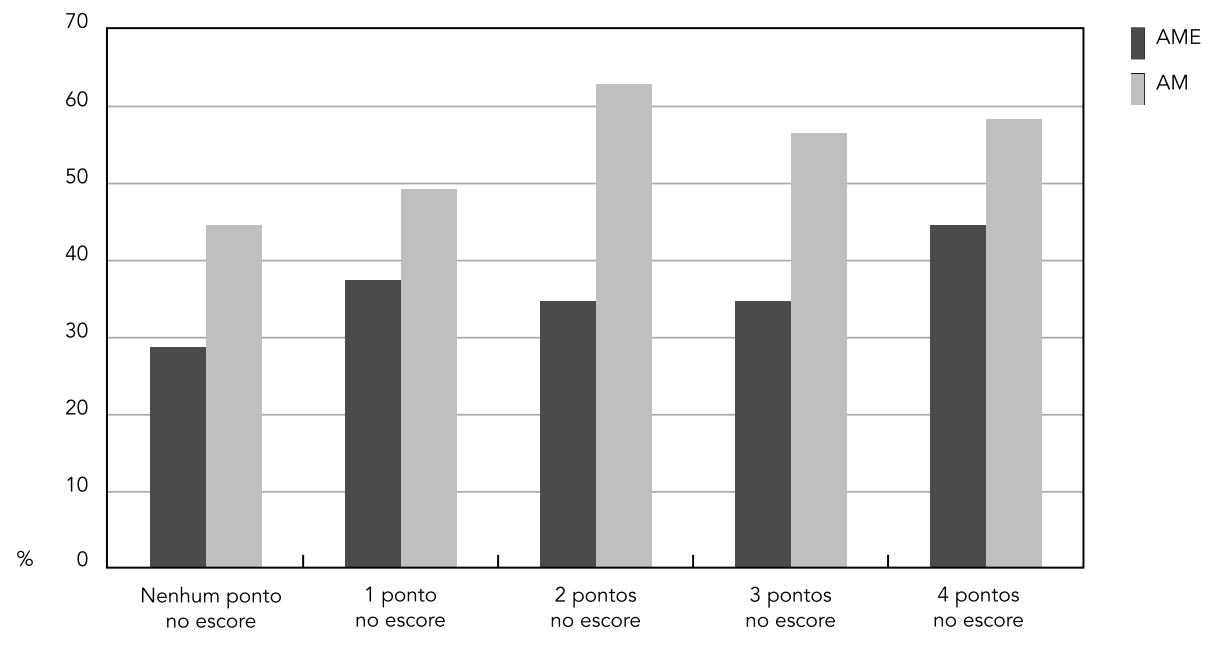

AM: aleitamento materno; AME: aleitamento materno exclusivo; UBS: unidades básicas de saúde.

que caracterizam a Rede Amamenta Brasil idealizados pelo Ministério da Saúde. Logo, a criação do escore de implantação da Rede mostrouse útil, pois se constatou que os indicadores de AM variam de acordo com o número de critérios cumpridos.

A atuação dos tutores, que poderia favorecer a implantação da Rede Amamenta Brasil, não aconteceu conforme as diretrizes da estratégia. Acreditamos que as dificuldades das UBS para cumprir os critérios de certificação na Rede poderiam ter sido minimizadas caso a proposta de acompanhamento pelos tutores houvesse sido concretizada. Visitas sistemáticas dos tutores às UBS são uma inovação da Rede Amamenta Brasil em relação a outras ações e programas, a qual encontra-se alinhada à discussão atual sobre a importância do apoio matricial às equipes de Atenção Básica 16. Na proposta formulada pelo Ministério da Saúde, os tutores da Rede Amamenta Brasil deveriam visitar regularmente as UBS, de preferência a cada três meses, para apoiar as equipes na implantação do plano de ação, elaboração e implantação do fluxograma de atendimento à dupla mãe-bebê e monitoramento dos indicadores. Chama a atenção que somente $12,5 \%$ das UBS em Dourados e 33,3\% em Porto Alegre recebiam visitas regulares dos tutores. Por outro lado, essa proposta mostrou- se plenamente viável no Município de Ribeirão Preto, onde $100 \%$ das UBS relataram visitas periódicas dos tutores (dados não apresentados).

As dificuldades de implantação da Rede Amamenta Brasil ficam evidentes quando se analisa a evolução da estratégia desde o seu lançamento. No período de 2008-2010, 58 oficinas haviam sido realizadas em todos os estados da federação, formando 1.415 tutores e envolvendo 1.034 UBS, além de 22.575 profissionais que atuavam na Atenção Básica. Todavia, somente em novembro de 2010, foram certificadas as primeiras UBS, sendo dez no Município de Corumbá (Mato Grosso do Sul) e oito em Dourados 11. Em relação às dificuldades de implantação da Rede Amamenta Brasil, cabe observar aspectos que mostraram influenciar o grau de implantação desta, tais como sua inserção no projeto de governo, a direcionalidade do projeto de implantação, a disponibilidade de recursos financeiros e o papel da coordenação da Rede no nível municipal, a fim de fortalecer a implantação da proposta 12 .

A prevalência da amamentação exclusiva em menores de seis meses, na amostra analisada, foi de $38 \%$, inferior à obtida na II Pesquisa de Prevalência do Aleitamento Materno nas Capitais Brasileiras e Distrito Federal, que foi de $41 \%$ em 2008 17. Tendo em vista que o AM é a estratégia que, isoladamente, tem maior impacto na 
redução da mortalidade infantil 18, é importante que nos municípios sejam intensificadas as ações para expandir essa prática.

Estudos sugerem que a prevalência da amamentação sofre influência não só das características das crianças e das mães, mas também da existência de programas e ações pró- amamentação nos municípios 19,20. Mais especificamente em relação ao impacto de intervenções realizadas no nível de Atenção Básica, estudos no Município do Rio de Janeiro evidenciam o papel do cuidado nesse nível de atenção sobre a prática da amamentação exclusiva ${ }^{21}$ e o impacto da Iniciativa Unidade Básica Amiga da Amamentação (IUBAAM) 22. Ainda em relação à IUBAAM, pesquisa realizada no Município de Montes Claros, Minas Gerais, constatou que, nos locais onde essa estratégia foi adotada, a duração de AME foi significantemente maior 23 .

Sobre o impacto da Rede Amamenta Brasil nas prevalências do AM, as pesquisas ainda são incipientes. Passanha et al. 24 analisaram, em um dos municípios incluídos neste estudo (Ribeirão Preto), crianças acompanhadas em serviços públicos certificados na Rede Amamenta Brasil ou que haviam realizado a oficina preconizada pela Rede e em serviços públicos que não aderiram à Rede Amamenta Brasil, além de serviços privados. A prevalência de amamentação exclusiva em menores de seis meses foi significativamente maior (razão de prevalência - RP $=1,41$; intervalo de $95 \%$ de confiança - IC95\%: 1,01;1,95) nas crianças acompanhadas em unidades de saúde certificadas na Rede, sugerindo que essa pode ser uma estratégia efetiva para aumentar os índices de AME. Por outro lado, estudo de impacto da
Rede Amamenta Brasil, desta vez em um município do Sul do Brasil, não encontrou diferença estatisticamente significativa nas prevalências de AME e de AM entre as crianças acompanhadas por serviços que aderiram à Rede Amamenta Brasil (certificados e não certificados) e as que frequentavam serviços que não foram expostos às ações da estratégia. Os autores atribuíram esse resultado às dificuldades encontradas na implementação da Rede no município avaliado, tais como descontinuidade do cumprimento dos critérios de certificação da Rede Amamenta Brasil das unidades já certificadas, baixo grau de cumprimento dos critérios pela maioria das unidades que iniciaram o processo de certificação na Rede, mas que ainda não haviam sido certificadas na época da coleta dos dados, alto grau de rotatividade dos profissionais de saúde e não utilização de protocolos de manejo do AM, inclusive nas unidades certificadas 25 .

Esses achados contraditórios reforçam a recomendação de realização de mais estudos de avaliação do impacto da Rede Amamenta Brasil, substituída pela Estratégia Amamenta e Alimenta Brasil, que explorem seu efeito, controlando a influência de outras variáveis individuais e contextuais. A Estratégia Amamenta e Alimenta Brasil é baseada no mesmo referencial teórico, e seu desenho de implementação guarda semelhança com aquele da Rede Amamenta Brasil. Sendo assim, espera-se que o presente estudo possa contribuir para o aprimoramento do processo de implantação dessa nova estratégia, com vistas à obtenção de melhores índices de amamentação e alimentação complementar no país.

\section{Colaboradores}

S. I. Venancio contribuiu no delineamento e concepção da pesquisa, elaboração do texto e revisão final. E. R. J. Giugliani colaborou no delineamento e concepção da pesquisa e revisão final do artigo. O. L. O. Silva, J. Stefanello, M. H. D’A. Benicio, M. C. G. Reis, L. C. E. Santo contribuíram no delineamento e concepção da pesquisa e revisão final do artigo. M. R. A. Cardoso participou na concepção da pesquisa, delineamento amostral e revisão final do artigo. G. S. Rios participou na concepção e execução da pesquisa e revisão final do artigo.

\section{Agradecimentos}

Às equipes da Coordenação Geral de Saúde da Criança e Aleitamento Materno do Ministério da Saúde e dos municípios de Dourados, Ribeirão Preto e Porto Alegre pelo apoio à realização deste estudo. 


\section{Referências}

1. Venancio SI, Saldiva SRDM, Monteiro CA. Tendência secular da amamentação no Brasil. Rev Saúde Pública 2013; 47:1205-8.

2. Departamento de Atenção Básica, Secretaria de Atenção à Saúde, Ministério da Saúde. Dez passos para uma alimentação saudável: guia alimentar para crianças menores de dois anos: um guia para o profissional da saúde na atenção básica. 2a Ed. Brasília: Ministério da Saúde; 2013.

3. Kramer MS, Chalmers B, Hodnett ED, Sevkovskaya Z, Dzikovich I, Shapiro S, et al. Promotion of Breastfeeding Intervention Trial (PROBIT): a randomized trial in the Republic of Belarus. JAMA 2001; 285:413-20.

4. Cattaneo A, Buzzetti R. Effect on rates of breast feeding of training for the Baby Friendly Hospital Initiative. BMJ 2001; 323:1358-62.

5. Guise JM, Palda V, Westhoff C, Chan BK, Helfand M, Lieu TA. The effectiveness of primary carebased interventions to promote breastfeeding: systematic evidence review and meta-analysis for the US Preventive Services Task Force. Ann Fam Med 2003; 1:70-8.

6. Renfrew MJ, Dyson L, Wallace L, D’Souza L, McCormick F, Spiby H. The effectiveness of public health interventions to promote the duration of breastfeeding. London: National Institute for Health and Clinical Excellence; 2005.

7. Coutinho SB, de Lira PI, de Carvalho Lima M, Ashworth A. Comparison of the effect of two systems for the promotion of exclusive breastfeeding. Lancet 2005; 366:1094-100.

8. Morrow AL, Guerrero ML, Shults J, Calva JJ, Lutter C, Bravo J, et al. Efficacy of homebased peer counselling to promote exclusive breastfeeding: a randomised controlled trial. Lancet 1999; 353:1226 31.

9. Pisacane A, Continisio GI, Aldinucci M, D'Amora S, Continisio P. A controlled trial of the father's role in breastfeeding promotion. Pediatrics 2005; 116:e494-8.

10. Rea MF. Reflexões sobre a amamentação no Brasil: de como passamos a 10 meses de duração. Cad Saúde Pública 2003; 19 Suppl 1:S37-45.

11. Área Técnica de Saúde da Criança e Aleitamento Materno, Secretaria de Atenção à Saúde, Ministério da Saúde. Rede Amamenta Brasil: primeiros passos (2007-2010). Brasília: Ministério da Saúde; 2011. (Série I História da Saúde).

12. Venâncio SI, Martins MCN, Sanches MTC, Almeida H, Rios GS, Frias PG. Análise de implantação da Rede Amamenta Brasil: desafios e perspectiva da promoção do aleitamento materno na atenção básica. Cad Saúde Pública 2013; 29:2261-74.

13. Cavalcante MGS, Samico I, Frias PG, Vidal AS. Análise de implantação das áreas estratégicas da atenção básica nas equipes de Saúde da Família em município de uma Região Metropolitana do Nordeste Brasileiro. Rev Bras Saúde Matern Infant 2006; 6:437-45.
14. Departamento de Ações Programáticas e Estratégicas, Secretaria de Atenção à Saúde, Ministério da Saúde. II Pesquisa de Prevalência de Aleitamento Materno em municípios brasileiros. Brasília: Ministério da Saúde; 2010.

15. Indicators for assessing infant and young child feeding practices. Part 1: definitions. Conclusions of a consensus meeting held 6-8 November 2007 in Washington D.C., USA. Geneva: World Health Organization; 2008.

16. Campos GWS, Domitti AC. Apoio matricial e equipe de referência: uma metodologia para gestão do trabalho interdisciplinar em saúde. Cad Saúde Pública 2007; 23:399-407.

17. Venancio SI, Escuder MML, Saldiva SRDM, Giugliani ERJ. Breastfeeding practice in the Brazilian capital cities and the Federal District: current status and advances. J Pediatr (Rio J.) 2010; 86:317-24.

18. Jones G, Steketee RW, Black RE, Bhutta ZA, Morris SS; Bellagio Child Survival Study Group. How many child deaths can we prevent this year? Lancet 2003 ; 362:65-71.

19. Venancio SI, Monteiro CA. Individual and contextual determinants of exclusive breast-feeding in São Paulo, Brazil: a multilevel analysis. Public Health Nutr 2006; 9:40-6.

20. Venancio SI, Saldiva SRDM, Escuder MML, Giugliani ERJ. The Baby-Friendly Hospital Initiative shows positive effects on breastfeeding indicators in Brazil. J Epidemiol Community Health; 2011; 66:914-8.

21. Pereira RS, Oliveira MI, Andrade CL, Santos Brito A. Fatores associados ao aleitamento materno exclusivo: o papel do cuidado na atenção básica. Cad Saúde Pública 2010; 26:2343-54.

22. Cardoso LO, Vicente AST, Damião JJ, Rito RVVF. Impacto da implementação da Iniciativa Unidade Básica Amiga da Amamentação nas prevalências de aleitamento materno e nos motivos de consulta em uma unidade básica de saúde. J Pediatr (Rio J.) 2008; 84:147-53.

23. Caldeira AP, Fagundes GC, Aguiar GN. Intervenção educacional em equipes do Programa de Saúde da Família para promoção da amamentação. Rev Saúde Pública 2008; 42:1027-33.

24. Passanha A, Benicio MHA, Venancio SI, Reis MCG. Implantação da Rede Amamenta Brasil e prevalência de aleitamento materno exclusivo. Rev Saúde Pública 2013; 47:1141-8.

25. Brandão DS, Venancio SI, Giugliani ERJ. Associação entre a implantação da Rede Amamenta Brasil e indicadores de aleitamento materno em um município do sul do Brasil. J Pediatr (Rio J.) 2015; 91:143-51. 


\section{Abstract}

This study aimed to evaluate the implementation of the Brazilian Breastfeeding Network and its impact on breastfeeding indicators. Implementation was analyzed according to type, including 56 primary healthcare units from three Brazilian municipalities. For evaluation of the degree of implementation, a score was created for each unit based on compliance with Ministry of Health certification criteria. Effects of implementation were analyzed according to exclusive and overall breastfeeding rates. Eighteen (32.1\%) of the units met the four criteria for certification. The study sample included 1,052 children less than one year of age, of whom 563 were less than six months old. Units that met the four criteria for certification showed higher rates of exclusive breastfeeding (44\%) when compared to the other units. Difficulties in implementing the Brazilian Breastfeeding Network were identified, and breastfeeding indicators varied according to the number of certification criteria met by the primary healthcare unit.

Breast Feeding; Primary Health Care; Public Health Policy

\section{Resumen}

El objetivo de este artículo fue evaluar la implantación de la Red Amamanta Brasil y su impacto sobre indicadores de lactancia materna (AM). Se realizó un análisis de implantación de segundo tipo, incluyendo 56 unidades básicas de salud (UBS) de tres municipios brasileños. Para la evaluación del grado de implantación se creó un marcador para cada UBS, basado en el cumplimento de los criterios de certificación del Ministerio de Salud; y para el análisis de los efectos de la implantación fueron investigadas las prevalencias de lactancia materna exclusiva y lactancia. Se verificó que 18 (32,1\%) UBS cumplían los cuatro criterios preconizados para certificación. Se incluyeron en el estudio 1.052 niños menores de un año, siendo 563 menores de seis meses. Se verificó que las UBS que cumplian los cuatro criterios de certificación tuvieron mayor prevalencia de lactancia materna exclusiva (44\%) cuando se comparan con las demás UBS. Dificultades para la implantación de la Red Amamanta Brasil fueron identificadas y los indicadores de AM variaron de acuerdo con el número de criterios de certificación cumplidos por las UBS.

Lactancia Materna; Atención Primaria de Salud; Políticas Públicas de Salud
Recebido em 27/Jan/2015

Versão final reapresentada em 30/Jun/2015 Aprovado em 23/Jul/2015 